\title{
THE TEMPERATURES OF WHITE DWARFS IN ACCRETING BINARIES
}

\author{
Paula Szkody \\ Department of Astronomy, University of Washington \\ Seattle, Washington 98195 \\ Edward M. Sion \\ Department of Astronomy and Astrophysics, Villanova University \\ Villanova, Pennsylvania 19085
}

\section{Introduction}

Through the use of accreting binary systems, it is possible to study the effects of the deposition of matter and energy on the surface of a white dwarf. The observed atmospheric properties of composition and temperature obtained from direct observation of the spectral lines and the continuum flux can be used to compare with those of single white dwarfs to understand the consequences of mass accretion on binary evolution.

Cataclysmic variables provide one of the best targets for this type of study because a) the primaries are all white dwarfs b) the level and the timescale of the accretion cover a large range from the high rate, relatively steady novalike accretors to the dwarf novae systems which are modulated on short timescales in a quasi-periodic manner. Unfortunately, due to the mass transfer process, an accretion disk builds up to the point where its radiation overwhelms the white dwarf light in most cases. Thus, to study the effects on the stellar primary, systems must be found which have low mass transfer rates (generally the short orbital period systems (Patterson 1984)) and/or high inclinations (since most of the disk flux emerges perpendicular to the plane of the disk). The best identification of the white dwarf emerges from IUE spectra which show a broad Lyman $\alpha$ absorption profile (in contrast to the normal emission lines from a disk at quiescence). The shape of this profile provides a sensitive indicator of the temperature and gravity. In some cases, broad absorption lines are also evident in the optical Balmer lines, although the broad emission lines from the disk usually make these difficult to detect. The steeply falling flux distribution of a white dwarf throughout the optical region, combined with a flat disk distribution usually means that the white dwarf contributes a minor amount to the optical flux. However, in the ultraviolet, the rising energy distribution of the white dwarf easily dominates the falling energy distribution of a low accretion rate disk (Mateo and Szkody 1984). White dwarfs are generally acknowledged to be prominent in the dwarf novae U Gem (Panek and Holm 1984), VW Hyi (Mateo and Szkody 1984) and Z Cha (Marsh, Horne and Shipman 1987) and suggested in EK TrA and WZ Sge (Verbunt 1987). In addition, the white dwarf has been seen in some novalike systems which sporadically turn off their mass transfer, (resulting in the disappearance of most of the disk and the resulting appearance of the white dwarf). This has been the case in TT Ari (Shafter et al. 1985) and 
some limits have been determined for MV Lyr (Szkody and Downes 1982) and V794 Aql (Szkody, Downes and Mateo 1988). Several magnetic white dwarfs have also been seen when the mass transfer ceases in the AM Her systems (summarized in Szkody, Downes and Mateo 1988).

The temperatures for the white dwarfs in cataclysmic variables (regarded as upper limits) range from $9000 \mathrm{~K}$ to more than $50000 \mathrm{~K}$, with a mean temperature of $25000 \mathrm{~K}$ compared to $12000 \mathrm{~K}$ for single white dwarfs (Sion 1986; 1987). In looking at the temperature as a function of the orbital period of the cataclysmic variables (Sion 1986,1987; Szkody, Downes, and Mateo 1988), it appears that there is a positive correlation in these parameters. If the orbital period is related to mass accretion rate as suggested by Patterson (1984), then the implication is that long term accretion has a heating effect on the underlying white dwarf.

To better understand the accretion interaction with the white dwarf, a few studies have been undertaken of the white dwarf following an outburst. This includes VW Hyi (Verbunt et al. 1987) and U Gem (Kiplinger, Sion and Szkody 1988). Both of these investigations have shown a decreasing ultraviolet flux after the optical flux has reached quiescence, which could be interpreted as a decreasing accretion rate or a cooling of the outer layers of the white dwarf following heating by the outburst. The indications from the U Gem study is that the white dwarf cools for several months. In both cases, the next outburst interrupted the monitoring sequence (after 14 days in VW Hyi and after 108 days in U Gem). Perhaps the best candidate for a long term study is the dwarf nova WZ Sge which has an outburst recurrence time of $33 \mathrm{yrs}$ and has a prominent white dwarf which is evident in the ultraviolet and optical. Many spectra have been obtained with IUE and exist in the archive following the 1978 outburst. Holm (1988) has reported on the results up through 1981 and Hassall (1987) reported on the spectrum through 1986. We report on the results through May 1988 in section II and discuss the general implications from all 3 systems in section III.

\section{WZ Sge}

This dwarf nova has very large amplitude outbursts at long intervals. The last outburst occurred on Dec. 1, 1978 when the system reached $V=7.8$. A light curve compiled by the AAVSO (Bortle 1979) shows that optical quiescence (V 15) was reached in April, 1979 (about 126 days after outburst). Holm (1988) shows IUE spectra from outburst (a flat disk distribution) through Nov. 23, 1981 (distribution similar to DA white dwarfs). He finds that the April 25, 1980 spectrum matches a $15400 \mathrm{~K}$ white dwarf, while the Nov. 23, 1981 spectrum matches a $14500 \mathrm{~K}$ white dwarf. We obtained additional SWP and LWP exposures on July 5, 1987 and May 1, 1988 and extracted exposures from the archive obtained on Nov. 3, 1986. We then extracted IUE spectra of DA white dwarfs of known temperature from the archive: 40 Eri B ( $T=16325)$, Wolf 485A $(T=14000)$ and $G 8-8(T=13000)$ for comparison. We then used the flux at $1500 \mathrm{~A}$ to normalize WZ Sge to each of the single white dwarfs and determine the best fit in temperature. Our results along with those of Holm (1988) are summarized in Table 1 and Figure 1 shows a representative fit of the May, 1988 spectrum of WZ Sge with Wolf 485A. 
TABLE 1 Temperature fits of SWP Spectra

\section{Date}

April 25, 1980

Nov. 23, 1981

Nov. 3, 1986

July 5,1987

May 1, 1988
1500A Flux

(x E-14)

9.0

6.5

2.0

3.5

2.2

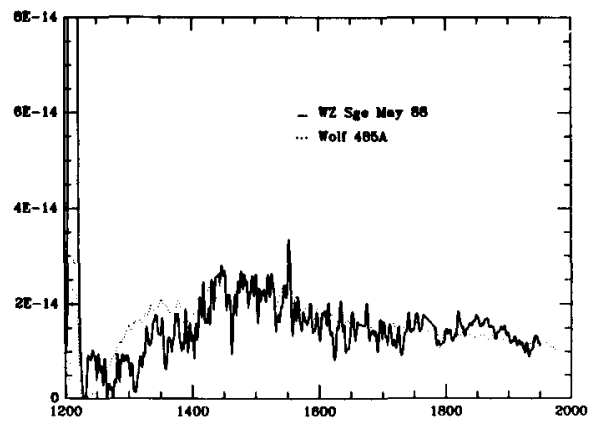

From these results, it appears that the UV flux reached some sort of equilibrium value about the end of 1986. The small excursions of brightness (as seen in July, 1987) may be fluctuations in the accretion rate (they cannot be due to orbital variations since the IUE exposures are all long i.e., $180 \mathrm{~min}$ SWP and $120 \mathrm{~min}$ LWP as compared to the binary orbit of $82 \mathrm{~min}$ ). This means that the UV flux continued to decrease for $8 \mathrm{yrs}$ after outburst (while the optical flux reached quiescence in 126 days). If the underlying white dwarf has provided a dominant amount of the UV flux since the disk reached quiescence (April, 1979), then the outer layers of the white dwarf cooled by several thousand degrees.

The question remains as to whether we are indeed viewing the white dwarf and what portion of it has been heated. Holm (1988) points out 2 possible problems: 1) if the April 1980 IUE flux is fit with a $15400 \mathrm{~K}$ white dwarf, then the optical flux would imply a V magnitude of 14.6, slightly above the normal quiescent value and 2) the equivalent widths of the absorption features do not change much between Jan. 1979 (outburst) and Nov. 1981. Point 1 cannot be proven true or false in terms of the observed values, because the AAVSO detection limits are near 14.6. To investigate Point 2, we measured the equivalent widths in the spectra after 1981.

Figure 1 shows that the May, 1988 spectra differ from Wolf $485 \mathrm{~A}$ primarily in the spectral region from 1250 to $1400 \mathrm{~A}$. This is the area of the Si II and III absorption lines, although it is difficult to measure the equivalent widths accurately due to the correct positioning of the continuum level in this area. Our measurements for the absorption lines are summarized along with Holm's measurement in Table 2. The values for U Gem (Kiplinger, Sion and Szkody 1988) are also listed for comparison.

TABLE 2 Equivalent Widths of Absorption Features

$\begin{array}{lccccc}\text { Date } & \text { Nov.81 } & \text { Nov.86 } & \text { July } 87 & \text { May } 88 & \text { U Gem } \\ \text { Si II } 1265 & 3.0 & 5.7 & - & 12.4 & 0.6 \\ \text { Si III } 1300 & 6.3 & 6.0 & 7.7 & 10.6 & 2.7 \\ \text { C II 1335 } & 3.9 & - & - & - & 0.8 \\ \text { Si IV } 1400^{*} & 5.4 & 4.6 & \text { hit } & 6.5 & 4.4 \\ \text { Si II } 1530 & 3.5 & 3.4 & 3.1 & 3.5 & -\end{array}$

*This feature may be the broad 1400A feature seen in single white dwarfs (Nelan and Wegner 1985) 
The SiII 1265 and Si III 1300 values are somewhat uncertain due to the poor signal in the continuum. These features also appear to be slightly redward of the correct wavelengths (Si II is at 1270 and 1535 , Si III at 1310) in the May 88 spectra (but this is probably an instrumental effect). However, it is apparent that there is an increase of the equivalent widths in Si II 1265 and SiIII 1300, consistent with an increasing contribution of a cooling white dwarf. The May 1988 equivalent width of $\mathrm{SiII}$ is consistent with a solar composition photospheric feature from a $13500 \mathrm{~K}$ white dwarf (Henry, Shipman and Wesemael 1985). The SiII 1530 feature might not show this effect due to the difficulties of measuring with some residual disk emission at CIV 1550 . For $\mathrm{T}<20,000 \mathrm{~K}$, the expected SiIV equivalent width in white dwarfs is less than $2 \mathrm{~A}$, so the measured absorption feature at $1400 \mathrm{~A}$ is most likely the $\mathrm{H}_{2}$ quasi-molecule (Wegner 1984; Nelan and Wegner 1985). If photospheric, this feature confirms that we are dealing with high density material $(\log g>6)$. Despite the fact that some accretion heating might be occurring, we expect this feature to maintain a similar equivalent width because it is more gravity than temperature sensitive.

To check the consistency of a $T=13500 \mathrm{~K}$ white dwarf dominating the quiescent flux of WZ Sge, we can compare the UV and optical fluxes from a white dwarf model (H. Shipman, private communication) with that of $\mathrm{WZ}$ Sge. The model gives a flux difference of a factor of 5.8 from 1300 to $5400 \mathrm{~A}$. Using the May 88 observed flux at $1300 \mathrm{~A}\left(8 \times 10^{-15}\right)$ results in a $\mathrm{V}$ mag of about 16. This is below the quiescent magnitude range of 15.2-15.5 and allows for some disk contribution, especially if the white dwarf does not contribute $100 \%$ of the $1300 \mathrm{~A}$ flux.

We can also determine the size of our proposed white dwarf via $\left(\mathrm{R}^{2}=\mathrm{Fd}^{2} / 4 \pi \mathrm{H}\right)$ and compare with estimates from optical solutions. For a $13500 \mathrm{~K}$ white dwarf, Shipman's models give $\mathrm{H}$ at $1300 \mathrm{~A}=5.5 \times 10^{7} \mathrm{ergs} / \mathrm{cm}^{2} / \mathrm{s} / \mathrm{A}$. Using a distance of $83 \mathrm{pc}$ for WZ Sge and the observed $1300 \mathrm{~A}$ flux, the corresponding radius comes out to be $8.7 \times 10^{8} \mathrm{~cm}$. The radial velocity solution of Gilliland, Kemper and Suntzeff (1986) results in a mass of 0.5 - 1.2 solar masses for the white dwarf. Our radius is consistent with the lower masses in this range, although Gilliland, Kemper and Suntzeff find that the secondary must be degenerate if the mass is low (for a main sequence secondary, the white dwarf mass must be near 1.1 solar masses, which would be a radius of $\left.5 \times 10^{8} \mathrm{~cm}\right)$.

In summary, the indications are that a $13500 \mathrm{~K} \pm 500 \mathrm{~K}$ white dwarf contributing most of the UV flux is consistent with the observed parameters of WZ Sge. However, the increase in temperature of this star to $15500 \mathrm{~K}$ is not enough to account for the factor of 4 increase in the $1500 \mathrm{~A}$ flux between 1988 and 1980 (Table 1). White dwarf models give a flux increase of about 3 for this temperature difference. Thus, there must also be some contribution from the hot disk or a larger emitting area to account for the April 1980 fluxes.

III. Summary

Table 3 summarizes the basic features of the 3 systems studied after accretion episodes. 
Table 3

\begin{tabular}{lrrrrlll} 
Object & $\begin{array}{r}\text { Orb. P } \\
(\mathrm{min})\end{array}$ & $\begin{array}{r}\text { Mass } \\
\text { WD }\end{array}$ & $\begin{array}{r}\text { Outburst } \\
\text { Interval }\end{array}$ & $\begin{array}{r}\text { WD T } \\
(\mathrm{K})\end{array}$ & $\begin{array}{l}\text { Opt.Min } \\
\text { Reached }\end{array}$ & $\begin{array}{l}\text { UV Min } \\
\text { Reached }\end{array}$ & $\begin{array}{l}\text { WD } \\
\text { Cooling }\end{array}$ \\
\hline VW Hyi & 107 & $(0.6)$ & $28 \mathrm{~d}$ & 18000 & $4 \mathrm{~d}$ & $>14 \mathrm{~d}$ & - \\
U Gem & 255 & 0.8 & $118 \mathrm{~d}$ & 30000 & $16 \mathrm{~d}$ & $>103 \mathrm{~d}$ & $40000-30000$ \\
WZ Sge & 82 & 1.1 & $33 \mathrm{yrs}$ & 13500 & $126 \mathrm{~d}$ & $2894 \mathrm{~d}$ & $15500-13500$
\end{tabular}

The data on these 3 cataclysmic variables support a dominant contribution from a white dwarf to the ultraviolet flux. The temperature of the white dwarf is a rising function of the orbital period of the system. After an outburst, presumably originating in increased accretion of solar composition material onto the white dwarf, the disk quickly returns to its quiescent emission levels (as evidenced by the rapid optical decline) but the UV flux takes a much longer time to return to its pre-outburst value. This slowly declining UV emission may represent the cooling of a white dwarf whose outer layers have been heated by the outburst. This is supported by the continuum distribution and the Lyman $\alpha$ profile fitting of the fluxes to DA white dwarfs. However, the total amount of flux change and the metallic absorption line fitting presents some difficulties with such a simple picture. In U Gem, a hotter area near the white dwarf must be invoked to account for the He II absorption lines seen. In WZ Sge, some disk component or larger area must also account for emission during part of the decline.

We gratefully acknowledge Harry Shipman for providing some fluxes for cool white dwarfs. NASA grant NSG 5395 provided support of this project.

Bortle, J. 1979, AAVSO Circ. 110.

Gilliland, R. C., Kemper, E. and Suntzeff, N. 1986, Ap. J., 301, 252.

Hassall, B. J. M. 1987, poster presentation at Aspen Workshop.

Henry, R. B. C., Shipman, H. L. and Wesemael, F. 1985, Ap. J. Suppl., 57, 145.

Holm, A. V. 1988, in A Decade of UV Astronomy with the IUE Satellite, ESA SP-281, in press. Kiplinger, A., Sion, E. M. and Szkody, P. 1988, Ap. J. submitted.

Marsh, T. R., Horne, K. D. and Shipman, H. L. 1987, M.N.R.A.S., 225, 551.

Mateo, M. and Szkody, P. 1984, A. J., 89, 863.

Nelan, E. P. and Wegner, G. 1985, Ap. J., 289, L31.

Panek, R. J. and Holm, A. V. 1984, Ap. J., 277, 700.

Patterson, J. 1984, Ap. J. Suppl., 54, 443.

Shafter, A. W., Szkody, P., Liebert, J., Penning, W. R., Bond, H. E. and Grauer, A. D. 1985, Ap. J., 290, 707 .

Sion, E. M. 1986, P.A.S.P., 98, 821.

Sion, E. M. 1987, I.A.U. Coll. 95, eds. A. G. D. Phillip, D. S. Hayes and J. Liebert, L. Davis press, p. 413.

Szkody, P. and Downes, R. A. 1982, P.A.S.P., 94, 328.

Szkody, P., Downes, R. A. and Mateo, M. 1988, P.A.S.P., 100, 362.

Verbunt, F. 1987, A. Ap. Suppl., 71, 339.

Verbunt, F., Hassall, B. J. M., Pringle, J. E., Warner, B. and Marang, F. 1987, M.N.R.A.S., 225, 113.

Wegner, G. 1984, A. J., 89, 1050. 